\title{
An Introduction to Lithography Machine
}

\author{
Xihao Zhang ${ }^{1, \mathrm{a}}$ \\ ${ }^{I}$ Collage of Safety Science and Engineering, Xi'an University of Science and Technology, Shannxi, Xi' an 710600, China \\ ${ }^{a}$ Email: lynnlin@xyzrgroup.com
}

\begin{abstract}
With the rapid advance of industrial intelligent manufacturing and electronic information technology, the importance of integrated circuits keeps increasing. As a fundamental equipment of integrated circuits manufacturing, the lithography machine has become a key research target for researchers around the world. This paper provides a short overview of the lithography technology and lithography machines. Key elements of lithography machines, such as the operation mode, the light source and photoresist, have been focused in this paper. The future development prospect of lithography technology is also summarized.
\end{abstract}

Keywords: Integrated circuit industry, Lithography machine, Light source, Operation mode.

\section{INTRODUCTION}

The integrated circuit industry is known as the modern "industrial food", leading the development of the future scientific and technological industrial revolution, driving the world's frontier technology innovation and development. The integrated circuit industry is a pioneering industry for social development, which mainly includes semiconductor materials, equipment, chip manufacturing, packaging testing, circuit design and other important links.

With the rapid advance of industrial intelligent manufacturing and electronic information technology, the importance of integrated circuits, especially the manufacturing of integrated circuits, keeps increasing.

Cross-disciplines is among the characteristics of integrated circuits manufacturing which is a collection of electronic science, computer science, communication technologies, machine manufacturing and some other multi-disciplinary disciplines. And the lithography machine is a typical representative of the interdisciplinary characteristic.

The integrated circuit industry mainly contains semiconductor materials, equipment, chip manufacturing, packaging and testing $[1,2]$, circuit design and other significant workflows[3]. Among that, semiconductor materials involve wafer material growth, special gases, particular chemical design and manufacturing; semiconductor equipment include lithography machines[4], etching machines, cleaning machines[5], and other processing and production equipment; the chip manufacturing refers to the organic combination of lithography[6], etching, thin film growth and other semiconductor processing processes to achieve the circuit design on the wafer; the package testing refers to the electrical connection and protection of manufactured chips . Meanwhile, it tests whether the function of chips qualify for the design requirements.

Besides, the circuit design achieves a certain function through the interconnection among components and devices, which has been achieved through EDA tools in modern large-scale integrated circuit design[7].

\section{LITHOGRAPHY}

Lithography is not only one of the most significant technologies in the production of chips, but also the most efficient high-precision micro processing technology in the world, which is supporting the realization and development of ultra-large-scale integrated circuits.

Lithography includes three important elements: the lithography process, photoresist and lithography machines[8]. During the production of chips, a wafer is covered with photoresist and put under the irradiation of a specific light source[9]. Then through the steps of development and etching, the complex graphics on the lithography mask are photocopied to the wafer which will be used for selective blocking precipitation or etching[10].

After exposure, a chemical reaction occurs in the exposed area, which allows the developer to dissolve part of the photoresist and exposes the wafer surface to obtain a specific lithographic pattern. According to the change 
of the film dissolution in the developer, photoresist can be divided into the positive photoresist and the negative photoresist [11]. Positive photoresists are soluble with a degradation reaction in the developer after exposure, while unexposed positive photoresists are insoluble. On the contrary, negative photoresists are insoluble in the developer because of the polymerization reaction under UV exposure, while the unexposed part is soluble in the developer.

\section{LITHOGRAPHY MACHINES}

The lithography machine has the name of "the crown jewelry of the semiconductor industry", and is the most complex, precise, and expensive equipment among all of the masterpieces of modern industry. The advanced lithography machines are generally divided into two types: the EUV lithography machine and the DUV lithography machine. And the DUV lithography machines are often divided into two types: the immersion type and the dry type. The principle of lithography technological originates from photographic plate making in printing technology, where micro graphs are formed by processing on a flat surface[12]. In fact, the lithography machine is the key equipment to realize the lithography process, which is used to copy the circuit structure on the mask to the silicon wafer.

With the development of science and technology, integrated circuits are developing towards high integration and excellent performances by reducing the chip size as much as possible. Therefore, increasing the resolution of lithography has become the core challenge for integrated circuits manufacturing. The theoretical resolution can be calculated using the Rayleigh equation:

$$
\mathrm{R}=\mathrm{k}_{1}(\lambda / \mathrm{NA})=\mathrm{k}_{1}[\lambda /(\mathrm{n} \cdot \sin \theta)]
$$

In the equation, the parameter of $\mathrm{R}$ is theoretical resolution; $\lambda$ is exposure wavelength ; NA is the numerical aperture; $\mathrm{k}$ is the process combination parameter; $\mathrm{n}$ is the refractive index of the medium; and $\theta$ is the minimum resolution angle.

From this equation, it is clear that increasing the theoretical resolution $\mathrm{R}$ of lithography can be achieved by shortening the wavelength $\mathrm{R}$ of the light source, increasing the numerical aperture NA and reducing the process combination parameter $\mathrm{k}_{1}$.

\section{MAIN COMPONENTS OF LITHOGRAPHY MACHINES}

The lithography machine is a complex structural equipment, and its main components include several major parts which consists of the laser light source, the objective lens system, the table system, the mask table system, the mask transfer system, the silicon transfer system, and the exposure system. The exposure system consists of projection objective lens and illumination system, which is the core of the lithography machine. There are several parts in the lithography machine, such as work table, mask table, mask transfer, and wafer transfer, serving as the execution system of the lithography machine, which are responsible for following the whole measurement and calibration process to achieve switching and precise positioning of different stations of silicon wafer and mask plate.

The process of the whole machine measurement and calibration relies on the measurement system of the lithography machine to achieve accurate measurement of each position of wafer and mask plate and the measurement system includes focus leveling, wafer alignment, mask alignment, interferometer, zero sensor, etc. Additionally, the equipment used to realize the calibration function of the whole system includes the beam corrector, energy controller, beam shape controller, etc. $[13,14]$.

\section{MAIN EXPOSURE MODES OF LITHOGRAPHY MACHINES}

According to different application scenarios, the mainstream lithography machines can be classified as the contact, proximity and projection type. These lithography machines all have different working principles and are suitable for different processing scenarios.

\subsection{The Contact Lithography Machine}

During the production process, a mask plate with a micro and nano graphic structure in a contact lithography machine comes into contact with each other. Under the irradiation of UV light, the graphics on the mask plate are transferred to the surface of the substrate to be processed. This kind of lithography machine is characterized by relatively simple equipment structure, high processing efficiency, low cost, easy realization of large chip area exposure, ease of use, long focal depth, and strong process adaptability. However, the process accuracy will be affected by the direct contact between the mask plate and photoresist contamination of the mask plate, mask plate wear, and some other problems. In addition, this kind of lithography machine has the disadvantage of low resolution, poor quality of exposed graphics, poor process consistency, etc. It can only realize the reproduction of mask plate graphics in equal proportion and cannot reduce the reproduction graphics.

\subsection{The Proximity Lithography Machine}

The proximity lithography machine is evolved from contact lithography machine. In the proximity lithography machine, there will be a tiny gap between the mask plate and the silicon wafer. Thus, the mask plate will not contact directly with the photoresist. Due to the separation, problems such as contamination of the mask plate by the photoresist can be effectively avoided, which 
improves the qualification rate of chip capacity and the production efficiency. At the same time, the service life of the mask plate is also extended.

However, in proximity lithography machine, the image received on the silicon wafer is not the geometric project in the mask plate, but the diffracted image of the mask plate as the gap between the silicon wafer and the mask causes diffraction effects. This directly affects the processing quality of the lithography process, resulting in distortion of the mask graphic edges.

\subsection{The Projection Lithography Machine}

The working principle of projection lithography is similar to that of a camera, in which the exposure between the mask plate and the photoresist is achieved by the optical pattern through an optical pattern. Since the projection lithography machine uses projection process, the mask and substrate are no longer in contact with each other, thus reducing the effect of mask plate defect. The project deflation of the optical system allows the processing of finer structures than the mask pattern, greatly improving the exposure resolution of the lithography process. Typically, the image of the mask plate is scaled by the projection of the optical system to one-fourth of its original size, thus enabling fine etching on the silicon wafer covered the photoresist. In addition, the projection lithograph machine has the exposure capability of scanning type imaging, which improves the productivity of the lithography process to some extent

\section{THE DEVELOPMENT OF LIGHT SOURCES}

During the development of lithography technology, people have been exploring light sources with shorter exposure wavelengths. From the 1980s to the early 1990s, the lithography process mainly used UV light sources with wavelengths of $436 \mathrm{~nm}$ (G-line) and 365nm (I-line) generated by high-pressure discharge mercury lamps as the exposure light sources for lithography, and the process node range was about $250-800 \mathrm{~nm}$. At the beginning of the 21 st century, researchers discovered that by refracting ArF light into ultrapure water, a light source with a wavelength of $134 \mathrm{~nm}$ could be obtained[15]. Eventually the lithography process reaches to 7-45 nm. With the increasing improvement of light source technology, the wavelength of the exposed light source is now developed to the extreme ultraviolet light source spectrum, and the wavelength reaches an amazing 13.5 $\mathrm{nm}$, which can realize the lithography process in the range of 3-7 $\mathrm{nm}$.

EUV lithography technology has been studied since the late 1980s. But the EUV light is easily absorbed due to its short wavelength and high energy which keeps being the bottleneck of EUV lithography technology. By continuously improving the collection device of EUV light, technicians finally make the UV light source meet the lithography demand.

At present, there are mainly four means to obtain the EUV light sources: Synchrotron Radiation Source (SRS) [16], Laser Produced Plasma (LPP) [17-19], Discharged Produced Plasma (DPP) and Laser-assisted Discharge Plasma (LDP) [20-25].Synchrotron radiation sources can produce high power EUV light sources and will not produce debris to contaminate the optical components. It can also output EUV light continuously. LPP, DPP and LDP all generate high temperatures of the target material by high energy beam, thus producing high temperature, high density plasma and emitting EUV light. Among them, LPP technology is to irradiate the target with high intensity pulsed laser as the driving energy, so that the target produces high temperature plasma and radiates EUV light; DPP is to cover the target between the anode and cathode, and the two electrodes produce strong discharge under high voltage, so that the target produces plasma, which is heated to produce EUV light; LDP is a combination of LPP and DPP technology, where the target is first irradiated with a pulsed laser to refine the target, then the DPP technology is used to discharge the target to produce EUV light[26].

\section{CONCLUSIONS}

With the development of integrated circuits, the lithography machine has been among the most sophisticated devices in the world and multiple kinds of lithography machines have been applied for the production of chips. In this review, typical lithography machines such as the contact, proximity, or projection lithography machine have been introduced. The main components and core systems, such as the objective lens system and mask transfer system, have also been reviewed. Key elements include the light source and photoresist for the lithography technology are included in this review as well.

As for the next generation of lithography machines, the technology of lithography will advance toward a higher exposure resolution, which can be achieved by continuously reducing the wavelength of the light source, the process parameters and increasing the numerical aperture according to the Rayleigh's equation. However, the difficulty of upgrading the technology of lithography and the manufacturing will increase meanwhile, and the cost will also increase dramatically. In fact, EUV lithography has already shortened the light source wavelength to $13.5 \mathrm{~nm}$, which leaves very limited opportunity to improve the resolution through the light source. In these circumstances, increasing the numerical aperture of the projection objective will provide an alternative approach.

A larger numerical aperture (NA) of the lithography indicates that the system is more capable of receiving 
diffracted light and is able to receive higher order diffraction levels. When a higher order of diffracted light is received, the image will achieve more details and higher resolution. The NA of the optical system used in the current EUV lithography is $0.33 \mathrm{NA}$, while ASML is developing the next generation EUV lithography machine with 0.55 NA. I If the technical bottleneck can be overcome, the process can be made to expand to $3 \mathrm{~nm}$.

Additionally, in order to further improve the exposure resolution, there are some other lithography technologies such as multiphoton lithography, surface plasma lithography, nanoimprinting, etc. These technologies may also be good candidates of next-generation lithograph.

\section{REFERENCES}

[1] Tellkamp, J.P., Thermal testing method for integrated circuit chips and packages. US, 2005.

[2] Koopman, N.G., et al., Method for testing, burn-in, and/or programming of integrated circuit chips. US, 1994.

[3] Baker, R.J., CMOS Circuit Design, Layout, and Simulation. 1997: CMOS Circuit Design, Layout, and Simulation.

[4] Nakayama, Y., Semiconductor equipment. 2005, US.

[5] Kilburn, J.I., Method and apparatus for cleaning semiconductor etching machines. US, 1993.

[6] Yamazaki, S., et al., Semiconductor chip and method for manufacturing the same. US, 2006.

[7] Deschamps, J.P., G.D. Sutter, and E. Cantó, EDA Tools. 2012.

[8] Wang Quanyong, S.B., Mai Yuliang, Progress of Photoresist and Its Film-forming Resin for Photoresist. Guangdong Chemical Industry, 2020. 47(14): p. 76-77.

[9] He Liwen, L.L., Meng Gang,Shao Jingzhen,Fang Xiaodong, Recent progress of novel photolithography technologies. Laser Technology, 2019. 43 : 30-37.

[10] Shao Wei, L.H., Challenges and solutions of lithography. Fine and Specialty Chemicals 2021. 29(04): p. 1-4.

[11] Xie Wen, L.J., Li Ping, A novel uv photoresist matrix resin and its photolithographic processes Imaging Science and Photochemistry, 2010. 28(01):52-58.

[12] Boru, F., The resolution limit of optical microlithography and its prospects Opyo-Electronic Engineering 1994. 021(002): p. 57-64.
[13] Tan Enmin, S.T., Zhang Zhigang,Zhang Lin, Adaptive feedforward control for reticle stage of lithography. Control Engineering of China, 2021. 28(06): p. 1069-1074.

[14] Hui, C., Design of the integrated Process for the Step-scan Lithography. China Integrated Circult, 2020. 29(Z4): p. 74-78.

[15] Levinson, H.J., Principles of lithography: Third edition. 2011.

[16] Mandai, et al., Development of compact synchrotron light source for x-ray lithography (abstract). Review of Scientific Instruments, 1989.

[17] Tatsumi, T., et al. Development progress update of the high power LPP-EUV light source using a magnetic field. in International Conference on Extreme Ultraviolet Lithography 2020. 2020.

[18] Mizoguchi, H., J. Fujimoto, and T. Saitou. LPP-EUV light source development for high volume manufacturing lithography. in SPIE Advanced Lithography. 2013.

[19] BRANDT, et al. Laser Produced Plasma Source System Development. in Emerging Lithographic Technologies XI. 2008.

[20] Zhu, Q., Plasma Dynamics in a $13.5 \mathrm{~nm}$ Laserassisted Discharge Plasma Extreme Ultraviolet Source.

[21] Thompson, K.C., et al., Experimental test chamber design for optics exposure testing and debris characterization of a xenon discharge produced plasma source for extreme ultraviolet lithography. Microelectronic Engineering, 2006. 83(3): p. 476484.

[22] Schriever, G., et al., Laser-produced plasma versus laser-assisted discharge plasma: physics and technology of extreme ultraviolet lithography light sources. Journal of Micro/ Nanolithography Mems \& Moems, 2012. 11(2): p. 021104.

[23] Katsuki, S., N. Tomimaru, and H. Akiyama, Study on laser-assisted discharge plasmas for efficient EUV emission. 電気学会研究会資料. ppt，パル スパワー研究会, 2008. 2008: p. 47-51.

[24] Hassanein, A., et al., HEIGHTS initial simulation of discharge produced plasma hydrodynamics and radiation transport for extreme ultraviolet lithography. Proceedings of SPIE - The International Society for Optical Engineering, 2003. 5037(1): p. 714-727.

[25] Borisov, V., et al., Discharge produced plasma source for EUV lithography. Proc Spie, 2007. 8322(3). 
[26] Zong Nan, H.W., Wang Zhimin,Wang Xiaojun,Zhang Shenjin,Bo Yong,Peng Qinjun,Xu Zuyan, Research progress on laser-produced plasma light source for $13.5 \mathrm{~nm}$ extreme ultraviolet lithography. Chinese Optics, 2020. 13(01): p. 28-42. 